\title{
Gemcabene Calcium
}

National Cancer Institute

\section{Source}

National Cancer Institute. Gemcabene Calcium. NCI Thesaurus. Code C75255.

A calcium salt formulation of gemcabene, a dialkyl ether dicarboxylic acid with antihyperlipidemic activity. 